\title{
Enxaqueca Crónica e Refratária: Como Diagnosticar e Tratar
}

\section{Chronic and Refractory Migraine: How to Diagnose and Treat}

\author{
Elsa PARREIRA ${ }^{1,2}$, Isabel LUZEIRO ${ }^{3,4}$, José Maria PEREIRA MONTEIRO $\rrbracket^{5}$ \\ Acta Med Port 2020 Nov;33(11):753-760 - https://doi.org/10.20344/amp.12004
}

\section{RESUMO}

A enxaqueca é uma cefaleia muito prevalente na população com importantes custos pessoais, sociais e económicos e é a segunda causa a nível mundial de anos vividos com incapacidade. As suas variantes, crónica (aquela que ocorre mais de 15 dias por mês) e refratária (resistente ao tratamento), sobretudo quando se complicam de uso excessivo de analgésicos, embora mais raras, constituem as formas que causam maior incapacidade. Os autores revêm estes três tipos de cefaleias (enxaqueca refratária, enxaqueca crónica e cefaleia secundária a utilização excessiva de analgésicos) que constituem um grupo de cefaleias de difícil terapêutica. São revistos a epidemiologia, os aspetos clínicos, os critérios de diagnóstico, as comorbilidades, os fatores de agravamento e o impacto destas cefaleias sobre a qualidade de vida dos doentes. O tratamento de cada uma destas entidades é abordado, ressalvando a importância de uma abordagem abrangente, considerando o tratamento das comorbilidades, a utilidade de medidas não farmacológicas, o imperativo de evitar o abuso de analgésicos e a necessidade absoluta de tratamento profilático. São revistos os diferentes tratamentos profiláticos disponíveis (e a evidência científica da sua eficácia), tais como os fármacos preventivos orais (neuromodeladores como o topiramato), a toxina botulínica tipo $\mathrm{A}$ e os novos medicamentos preventivos para a enxaqueca (anticorpos monoclonais que atuam sobre o péptido relacionado com o gene da calcitonina ou o seu recetor, e que são os primeiros medicamentos preventivos desenvolvidos especificamente para atuar na fisiopatogenia da enxaqueca. Para os casos refratários são consideradas outras alternativas terapêuticas como a neuroestimulação.

Palavras-chave: Perturbações da Cefaleia Secundários/induzido quimicamente; Perturbações de Enxaqueca/diagnóstico; Perturbações de Enxaqueca/tratamento farmacológico

\section{ABSTRACT}

Migraine is highly prevalent and carries a significant personal, social and economic burden. It is the second cause of disability (years living with disability) worldwide and the first cause under 50 years of age. Chronic migraine (occurring for more than 15 days a month) and refractory migraine (treatment resistant), especially when there is also analgesic overuse, are the most disabling forms of migraine. These three disorders (chronic migraine, refractory migraine and medication overuse headache) are particularly difficult to treat. This article reviews their epidemiology, clinical presentation, diagnostic criteria, risk factors, comorbidities and social and personal impact. The therapeutic options available are discussed and focused on a multidisciplinary approach, non-pharmacological interventions treatment of comorbidities and avoiding analgesic overuse. Prophylactic treatments are mandatory and include the oral prophylactic treatments (topiramate), botulinum toxin type A and the novel monoclonal antibodies against calcitonin gene related peptide or its receptor, which are the first migraine preventive medicines developed specifically to target migraine pathogenesis. In refractory cases, multiple therapies are required including neurostimulation.

Keywords: Headache Disorders, Secondary/chemically induced; Migraine Disorders/diagnosis; Migraine Disorders/drug therapy

\section{INTRODUÇÃO}

A enxaqueca é uma cefaleia primária muito prevalente, caracterizada por crises de dor de cabeça acompanhadas de náuseas, vómitos e intolerância a estímulos sensoriais.

Numa revisão recente de todos os estudos populacionais publicados, ${ }^{1}$ com uma amostra de seis milhões de pessoas, evidenciou-se uma prevalência de enxaqueca na população mundial de $11,6 \%$. Nos estudos de base clínica ou de coorte a prevalência de enxaqueca ao longo da vida é superior: cerca de $15 \%$. $^{2,3}$ Segundo a Organização Mundial de Saúde (OMS), ${ }^{4}$ a enxaqueca é a sexta doença mais prevalente a nível mundial afetando cerca de 1 a 1,09 biliões de pessoas.

A enxaqueca é mais frequente na mulher e tem habitualmente início na segunda década de vida, com um pico de prevalência entre a segunda e a quarta décadas.

Manifesta-se por crises de cefaleia que surgem com frequência variável. A dor é tipicamente pulsátil, de intensidade moderada a severa, durando até 72 horas. As cefaleias acompanham-se de: náuseas, vómitos, fotofobia, fonofobia, cinesiofobia (intolerância aos movimentos da cabeça), osmofobia (intolerância a odores), tonturas e vertigens. A sintomatologia agrava com os movimentos da cabeça e atividade física de rotina (como subir escadas ou apenas andar), obrigando ao repouso. Numa percentagem significativa são também referidos, durante a crise, sintomas cognitivos. . $^{5}$

Durante a crise, os doentes têm muitas vezes de interromper a sua atividade. Num estudo realizado nos Estados

\footnotetext{
1. Consulta de Cefaleias. Hospital Prof. Doutor Fernando Fonseca. Amadora. Portugal.

2. Centro de Cefaleias. Hospital da Luz. Lisboa. Portugal.

3. Consulta de Cefaleias. Centro Hospitalar Universitário de Coimbra. Coimbra. Portugal.

4. Departamento de Neurofisiologia. Escola Superior de Tecnologia da Saúde de Coimbra. Coimbra. Portugal.

5. Instituto de Biologia Molecular e Celular/Instituto de Investigação e Inovação em Saúde. Universidade do Porto. Porto. Portugal

$\triangle$ Autor correspondente: José Maria Pereira Monteiro. jmpereiramonteiro@gmail.com

Recebido: 01 de março de 2019 - Aceite: 06 de maio de 2019 | Copyright @ Ordem dos Médicos 2020
} 
Unidos da América (EUA), ${ }^{7}$ cerca de $54 \%$ dos entrevistados apresentavam incapacidade significativa nas crises, tendo obrigatoriamente de repousar no leito, e apenas $7 \%$ conseguiam manter atividade e rendimento normal.

É um importante problema de saúde pública; em 2017, a OMS considerou a enxaqueca como a segunda causa a nível mundial de anos vividos com incapacidade, sendo a primeira causa de incapacidade entre os 18 e os 50 anos. ${ }^{8}$

Classifica-se, consoante a frequência das crises, em episódica (a mais comum) - quando as crises têm uma frequência inferior a 15 dias por mês e alternam com períodos livres de sintomas - e crónica - quando as dores surgem mais de 15 dias por mês, muitas vezes diárias e contínuas.

A enxaqueca crónica (que ocorre mais de 15 dias por mês) e a enxaqueca refratária (resistente à terapêutica), sobretudo quando complicada por cefaleia por uso excessivo de medicação são as variantes de enxaqueca de mais difícil tratamento que serão abordadas neste artigo.

\section{ENXAQUECA CRÓNICA}

\section{Epidemiologia, fatores de risco e quadro clínico}

A variante de enxaqueca que maior incapacidade provoca é a enxaqueca crónica. Encontra-se definida na Classificação Internacional de Cefaleias (ICHD-3) (Tabela 1) como a cefaleia que dura mais de 15 dias por mês, durante três meses, e em que a cefaleia é típica de enxaqueca em pelo menos oito dias. ${ }^{9}$

É menos frequente que a enxaqueca episódica, com uma prevalência estimada de $1,0 \%$ a $2,4 \%$ da população adulta mundial, ${ }^{10,11} 8 \%$ do total de enxaquecas. ${ }^{10}$

Resulta habitualmente do agravamento progressivo da enxaqueca episódica, em que as crises se vão tornando mais frequentes e mais duradouras ao longo de meses ou anos. Esta cronificação estima-se que ocorra ao ritmo de cerca de $2,5 \%$ ao ano, ${ }^{12}$ mas num terço dos casos poderá ser rápida.

À medida que as crises aumentam de frequência vão perdendo algumas características de enxaqueca: passam a ser menos intensas, mais duradoras, com menos náuseas ou vómitos e sem outros sintomas acompanhantes. No entanto, o doente mantém intercaladas crises com características típicas de enxaqueca.

Estudos epidemiológicos identificaram fatores de risco que aumentam a probabilidade de cronificação ${ }^{7,13}$ (Tabela 2): 1. frequência basal das crises, se superior a oito dias por mês; 2. utilização demasiado frequente de medicação para tratamento agudo das crises; 3 . resposta insuficiente à terapêutica preventiva.

Alguns desses fatores de risco são modificáveis, tais como obesidade, sedentarismo, perturbações do sono e abuso de cafeína; outros não o são, como por exemplo a idade ou o género (duas a seis vezes mais frequente nas mulheres). ${ }^{7,14}$

A enxaqueca crónica acompanha-se de elevada percentagem de comorbilidades ${ }^{7}$ (Tabela 3 ) que devem ser tratadas concomitantemente porque geralmente agravam o quadro de enxaqueca. Salientam-se as perturbações do sono, as perturbações psiquiátricas e outras dores crónicas. ${ }^{15,16}$

O impacto económico e social da enxaqueca crónica é enorme. Comparando com as formas episódicas, estes doentes apresentam menores rendimentos, menor probabilidade de terem emprego a tempo inteiro, maior incapacidade, menor qualidade de vida, maiores níveis de ansiedade

Tabela 1 - Critérios de diagnóstico de enxaqueca crónica. International Classification of Headache Disorders (ICHD-3)

A. Cefaleia (idêntica à cefaleia de tipo tensão e/ou à da enxaqueca) em $\geq 15$ dias por mês, durante $>3$ meses e preenchendo os critérios B e C

B. Ocorrendo em um doente que tenha tido, pelo menos cinco episódios, preenchendo os critérios B-D de 1.1 Enxaqueca sem aura e/ou critérios $\mathrm{B}$ e $\mathrm{C}$ de 1.2 Enxaqueca com aura

C. Em $\geq 8$ dias por mês durante $>3$ meses, cumprindo algum dos seguintes:

1. critérios $C$ e $D$ de 1.1 Enxaqueca sem aura

2. critérios $B$ e $C$ de 1.2 Enxaqueca com aura

3. convicção do doente de que é enxaqueca no início e aliviada por um triptano ou ergotamínico

D. Não melhor explicada por outro diagnóstico da ICHD-3.

Tabela 2 - Fatores de risco para evolução para enxaqueca crónica (cronificação) 7,13,14,16

\begin{tabular}{ll}
\hline Não modificáveis & Modificáveis \\
\hline Idade & Utilização excessiva de analgésicos \\
Género Feminino & Abuso de cafeína \\
Baixo nível socioeconómico e de escolaridade & Frequência basal elevada de crises \\
Antecedentes de traumatismo craniano & Obesidade \\
Eventos traumáticos de vida & Sedentarismo \\
Má resposta à terapêutica preventiva & Perturbações do sono \\
& Perturbações psiquiátricas \\
& Doenças reumáticas \\
\hline
\end{tabular}


Tabela 3 - Comorbilidades da enxaqueca crónica ${ }^{7,12,15,16}$

\begin{tabular}{lll}
\hline Doenças Psiquiátricas & Patologia do Sono & Outras Dores Crónicas \\
\hline Ansiedade generalizada & Roncopatia & Fibromialgia \\
Depressão & Fragmentação do sono & Lombalgia \\
Doença nipolar & Insónia crónica & Disfunção articulação temporo-mandibular \\
Perturbação de pânico & Apneia obstrutiva do sono & \\
Perturbação obsessivo-compulsiva & Movimentos periódicos do sono & \\
Fobias & Bruxismo noturno & \\
& & \\
\hline Doenças Metabólicas & Doenças Reumáticas e Autoimunes & Outras \\
\hline Obesidade & Artrtite reumatóide & Colon irritável \\
Hipotroidismo & Lupus & Asma \\
& Síndrome do anticorpo antifosfolípido & Urticária crónica \\
& & Hipertensão arterial \\
& & Doença pulmonar obstrutiva crónica \\
& & Enfisema \\
\hline
\end{tabular}

e depressão e recorrem mais aos cuidados de saúde. ${ }^{12,17,18}$ Atingindo mais de $1 \%$ da população mundial, tem significativos custos económicos e sociais globais. Os custos diretos $(10 \%-20 \%$ dos custos totais) referentes ao consumo dos cuidados de saúde é 4,4 vezes maior na enxaqueca crónica que na episódica (US\$7750 vs US\$1757 / ano/doente nos EUA). ${ }^{18}$ Os custos indiretos decorrem da perda de produtividade: absentismo laboral ou escolar, diminuição da capacidade produtiva (presentismo), falhas a eventos familiares e sociais e menor assistência à família que os doentes com enxaqueca episódica. ${ }^{19}$

\section{Fisiopatogenia}

Admite-se que a repetição das crises de enxaqueca com estimulação repetida da via trigeminovascular leva a alteração funcional com sensibilização periférica e central (originando alodínia) e mesmo à produção de alterações estruturais. Estas surgem nas áreas cerebrais do processamento da dor (alterações da espessura do córtex, por exemplo); são detetáveis nos exames de neuroimagem; não ocorrem nas formas episódicas, surgindo apenas nos casos de longa evolução; e provavelmente são comuns noutras dores crónicas. ${ }^{20-22}$ Verificou-se também elevação sustentada de neuromediadores de ativação dos sistemas trigémino-vasculares como o péptido relacionado com o gene da calcitonina (CGRP) e o péptido intestinal vasoativo (VIP). ${ }^{23}$

\section{Diagnóstico diferencial}

O diagnóstico diferencial da enxaqueca crónica faz-se com outras cefaleias diárias. O doente fornece habitualmente uma história com anos de evolução de enxaqueca episódica típica e agravamento progressivo. Contudo, nos casos em que esta progressão não é clara, e sobretudo de instalação recente, devemos excluir causas secundárias. A mais frequente é a cefaleia secundária ao uso excessivo de medicação analgésica para tratamento da enxaqueca. Devem igualmente ser consideradas outras possibilidades, ${ }^{24}$ tais como a cefaleia secundária a patologia da coluna cervical, a disfunção temporo-mandibular ou a síndrome de apneia obstrutiva de sono (SAOS), sobretudo se de apresentação matinal. Não se deverá esquecer também a arterite de células gigantes ou a hipertensão intracraniana idiopática benigna.

A enxaqueca crónica faz também diagnóstico diferencial com cefaleias primárias crónicas, sobretudo a cefaleia tipo tensão crónica, de diferenciação extremamente difícil porque a enxaqueca crónica pode assemelhar-se à cefaleia de tensão. Esta última é sentida como uma dor ligeira bifrontal, de tipo peso ou moinha, e não se acompanha de outros sintomas, com exceção de fonofobia ou fotofobia e náuseas ligeiras. Não é agravada pelo esforço físico de rotina e não impede as atividades diárias.

\section{Tratamento}

O tratamento da enxaqueca crónica é difícil, multifatorial, desafiante e necessariamente persistente. O objetivo é reduzir a frequência e intensidade das crises, e melhorar a qualidade de vida.

Assenta em quatro pilares: 1) identificação e controlo dos fatores de cronificação; 2) prevenção ou tratamento do uso excessivo de analgésicos; 3) tratamento das comorbilidades; 4) terapêutica preventiva precoce e agressiva de modo a evitar o agravamento e permitir a conversão para enxaqueca episódica.

É necessário gerir as expectativas dos doentes e fazer um acompanhamento frequente. Deve ser explicada a natureza da sua doença, bem como o mecanismo que leva ao aparecimento da cefaleia por uso excessivo de medicação, e a absoluta necessidade da terapêutica preventiva. Aconselhar sobre o estilo de vida, com prática de exercício físico, dieta saudável, horários regulares de sono e evitar o 
abuso de cafeína.

É importante identificar os fatores desencadeantes das crises, sendo os mais frequentes ${ }^{17,25}$ : stress, menstruação, jejum prolongado e variações do ritmo de sono.

O tratamento não farmacológico deve ser oferecido a todos os doentes, nomeadamente técnicas cognitivo-comportamentais, de biofeedback, e treinos de relaxamento para controle do stress, com evidência de eficácia para as duas primeiras. ${ }^{26}$

O tratamento das comorbilidades e fatores de cronificação é fundamental. Poderá ser necessário o acompanhamento psicológico ou psiquiátrico.

Estas medidas, embora importantes, não serão suficientes na maioria dos doentes, que terão indicação para realização de terapêutica preventiva.

Dos vários fármacos que se utilizam eficazmente na enxaqueca episódica, ${ }^{27}$ tais como antiepiléticos (valproato, topiramato), amitriptilina, propranolol e flunarizina, apenas o topiramato e o valproato foram ensaiados especificamente na enxaqueca crónica com resultados positivos. Os estudos com beta-bloqueantes foram negativos. $\mathrm{O}$ valproato, no entanto, foi testado apenas num ensaio clínico com 29 doentes. ${ }^{28} \mathrm{O}$ topiramato evidenciou resultados positivos em dois ensaios clínicos randomizados e duplamente cegos com doses médias de 100 mg. ${ }^{29,30}$ É um fármaco neuromodelador, eficaz na redução do número de dias por mês com enxaqueca, com melhoria da qualidade de vida. Tem a desvantagem de, em cerca de $30 \%$ dos doentes, desenvolver efeitos secundários: sonolência, lentificação psicomotora, defeito de memória verbal, irritabilidade, parestesias, anorexia, litíase renal entre outros. Ao fim de um ano, apenas $20 \%$ dos doentes mantém a terapêutica profilática. ${ }^{31}$

Nos casos que não respondem ao topiramato ou outros fármacos profiláticos, está indicado o tratamento com toxina botulínica do tipo A. A sua eficácia foi estabelecida em dois ensaios clínicos, onde foram incluídos mais de 1300 doentes. ${ }^{32}$ Este tratamento consiste na aplicação, a cada três meses, de $5 \mathrm{U}$ de toxina botulínica por via subcutânea, em 31 locais do couro cabeludo previamente definidos (segundo o protocolo PREEMPT). É bem tolerado, com ligeiros efeitos secundários: desconforto mínimo na aplicação, ptose palpebral, rigidez ou dor cervical. Reduz o número de dias de enxaqueca, o número de dias de cefaleia intensa, o consumo de analgésicos, idas ao serviço de urgência e incapacidade resultante.

Recentemente, foi desenvolvido um grupo de fármacos inovadores para prevenção de enxaqueca que atuam sobre o CGRP, neuropeptideo implicado na patogénese da enxaqueca. O CGRP aumenta durante a crise de enxaqueca, e a sua administração desencadeia uma crise. ${ }^{33}$ Os níveis séricos de CGRP estão também aumentados na enxaqueca crónica. ${ }^{23}$ Estes fármacos, desenvolvidos especificamente para a profilaxia da enxaqueca, são anticorpos monoclonais. ${ }^{33-34}$ Existem três anticorpos anti-CGRP ou ligando do CGRP: fremanezumab, eptinezumab e galcanezumab, bem como um anticorpo antirecetor do CGRP, o erenumab. Tanto o erenumab como o galcanezumab obtiveram, para além do parecer positivo por parte do Committee for Medicinal Products for Human Use (CHMP) da Agência Europeia do Medicamento (EMA), autorização para introdução no mercado por parte da Comissão Europeia (na enxaqueca com mais de quatro dias por mês). O erenumab e o fremanezumab estão já a ser comercializados em Portugal.

O fremanezumab obteve parecer positivo pelo mesmo comité da EMA em janeiro de 2019. ${ }^{34}$ Todos são de administração parentérica, por via subcutânea (exceto o eptinezumab que é de administração endovenosa) e periodicidade mensal ou trimestral (como no caso do fremanezumab). ${ }^{34} \mathrm{O}$ seu efeito foi demonstrado de forma robusta e consistente em diversos ensaios clínicos $^{34-36}$ de fase II e III: redução sustentada do número de dias de cefaleias por mês e da necessidade de consumo de analgésicos, melhoria da incapacidade e da qualidade de vida. São eficazes na enxaqueca com e sem aura, episódica, frequente e crónica, mesmo em doentes que falharam previamente dois a quatro fármacos profiláticos ou que apresentavam abuso de analgésicos. ${ }^{34}$

Têm um perfil de segurança e de efeitos adversos muito favorável, com uma incidência de efeitos secundários praticamente semelhante à do placebo, entre eles obstipação, queixas nasofaríngeas ou reações no local da injeção. ${ }^{35}$ Outra vantagem é o seu rápido início de ação, logo nas primeiras semanas de tratamento, ao contrário do que sucede com os fármacos profiláticos de administração por via oral e com a toxina botulínica. ${ }^{35,36}$

No entanto, a experiência clínica com estes fármacos é ainda reduzida. Só uma ampla utilização permitirá confirmar o perfil de segurança favorável em populações especiais (com fatores de risco para doença cardiovascular) no que concerne ao risco do bloqueio crónico de um potente vasodilatador como o CGRP em eventos cardiovasculares agudos. $^{37,38}$

\section{Prognóstico}

Em cerca de $40 \%$ dos doentes a enxaqueca crónica persiste a longo prazo, em $26 \%$ reverte para episódica ao fim de dois anos e em 40\% alternam continuadamente entre as formas crónicas e episódicas, ${ }^{39}$ pelo que se espera que os novos fármacos preventivos possam vir a alterar este preocupante panorama clínico.

\section{CEFALEIA POR USO EXCESSIVO DE MEDICAÇÃO (MOH)}

Atualmente integradas no grupo de cefaleias secundárias da Classificação Internacional de Cefaleias (ICHD3, grupo 8$)^{9}$ as $\mathrm{MOH}$ são cefaleias que ocorrem em 15 ou mais dias por mês, em doentes com cefaleia primária pré-existente, devido ao uso excessivo e regular de medicação aguda ou sintomática (10 ou mais, ou 15 ou mais dias por mês, dependendo do fármaco), num período superior a três meses.

A MOH afeta $1 \%-2 \%$ da população $0^{40}$ num rácio muIher/homem de 3,5/1 e na quinta década. ${ }^{41} \mathrm{Em}$ número absoluto, cerca de 63 milhões de pessoas são afetadas. 
$\mathrm{Na}$ consulta terciária, a percentagem de doentes com $\mathrm{MOH}$ é de $30 \%-50 \%$.

Os custos com $\mathrm{MOH}$ rondam os $€ 3561$, três vezes os custos da enxaqueca. ${ }^{42}$ Este valor reflete os gastos com medicação, consultas e exames complementares, e a diminuição da produtividade laboral.

$\mathrm{A} \mathrm{MOH}$ é uma causa frequente da transformação de enxaqueca episódica em crónica: a toma do fármaco abortivo alivia a dor, mas após o efeito medicamentoso a cefaleia retorna, e assim sucessivamente, desenvolvendo-se dependência em relação ao medicamento e agravamento da cefaleia primária. ${ }^{43}$ Mais de $65 \%$ das pessoas tem um comportamento aditivo. Os fármacos em causa são todos os utilizados para tratar as crises de enxaqueca: Anti-inflamatórios não esteróides (AINEs), triptanos, analgésicos simples ou combinados. Os medicamentos combinados, os barbitúricos e os que contém opióides desenvolvem mais rapidamente dependência. $O$ facto do comprimido não abortar a crise não implica a cessação da toma, porque os doentes mantêm as tomas diariamente ou quase diariamente. .44, $^{45}$

$\mathrm{O}$ aparecimento da $\mathrm{MOH}$ depende da personalidade do doente, do limiar de tolerância à dor, do número prévio de crises mensais, da facilidade com que podem recorrer à medicação. Determinadas comorbilidades, tais como distúrbios gastrointestinais e mialgias, estão associadas à $\mathrm{MOH}{ }^{39}$ São reconhecidos como fatores de risco o baixo nível socioeconómico dos doentes, o sexo feminino e distúrbios do foro psiquiátrico. ${ }^{46,47} \mathrm{O}$ principal fator de risco foi identificado como sendo a existência prévia de enxaqueca, cefaleia de tensão, ou cefaleia em salvas.

Quando a cefaleia se perpetua, há facilitação da passagem do estímulo dor pela porta de controlo do tronco cerebral, e o estímulo chega com muito mais frequência às zonas da dor, que se vão amplificando. Os sistemas supra segmentares de controlo da dor deixam de funcionar. Cria-se um estado permissivo à dor. Os níveis de serotonina estão diminuídos, ${ }^{48}$ e os de CGRP e substância P aumentados. O sistema endocanabinóide está alterado, com diminuição de endorfinas e aceleração da degradação das mesmas, induzindo a dependência. ${ }^{49}$

Doentes com $\mathrm{MOH}$ e enxaqueca apresentam alterações nas áreas relacionadas com a dor, quer a nível cortical quer a nível do tronco cerebral. Estudos com ressonância magnética nuclear funcional (fRMN) revelaram diminuição da atividade do girus supramarginal e do córtex parietal superior e inferior, que normalizou seis meses após desintoxicação, ${ }^{50,51}$ e aumento do volume da substância cinzenta periaquedutal, importante no controlo descendente da dor. ${ }^{52}$

Habitualmente, a cefaleia desaparece após a paragem do excesso medicamentoso, mas não sistematicamente. O modo de tratamento deve ser ajustado a cada doente, às suas comorbilidades e às suas expectativas. A primeira medida é a suspensão dos fármacos em abuso, preferencialmente de modo abrupto, dependendo dos fármacos em causa. Nos primeiros 4 - 10 dias a dor pode agravar e, depois, paulatinamente, vai ocorrendo alívio. ${ }^{53} \mathrm{Em}$ alguns casos o doente apresenta sinais francos de dependência: vómitos, taquicardia, distúrbios do sono, inquietude, ansiedade e convulsões. ${ }^{43}$ Nestes casos, a hidratação endovenosa é a ação inicial, podendo seguir-se outras em função da sintomatologia (neurolépticos, terapia anticonvulsiva...). No caso de doentes com comportamento aditivo a programação do tratamento deve ser muito cuidadosa e com seguimento muito frequente.

Duração do abuso superior a um ano, combinação de fármacos, uso de opióides, doenças psiquiátricas concomitantes, e recorrência em tentativas anteriores, aumentam a suscetibilidade a complicações e associam-se ao insucesso do tratamento ${ }^{54}$.

É essencial identificaros grupos de risco, esclarecer os doentes e realizar acções de tratamento preventivo.

Medicação profilática pode ser iniciada em simultâneo com a suspensão dos analgésicos ou posteriormente, dependendo da experiência do médico e dos fatores associados. ${ }^{53}$

O risco de recorrência é muito elevado. Embora $50 \%$ a $60 \%$ melhorem com o tratamento, a taxa de recorrência aos seis anos, é de $40 \%-50 \% .{ }^{55}$ Sem mudança do estilo de vida e a implementação de mecanismos de adaptação para resolver o problema da dor, o comportamento mantém-se e a cefaleia reaparece. Trata-se de uma dependência e tem que ser encarada como tal.

\section{ENXAQUECA REFRATÁRIA}

Alguns doentes com enxaqueca, com ou sem aura, podem sentir ao longo do tempo um agravamento progressivo, em severidade e frequência das crises, e uma diminuição ou perda de resposta aos diversos tratamentos adequados. Considera-se como refratária ${ }^{56,57}$ qualquer forma de enxaqueca que não responda a duas de quatro classes de medicamentos profiláticos nem aos medicamentos da fase aguda (triptanos, dihidroergotamina, AINEs ou combinações de analgésicos $)^{9,56,57}$ administrados em doses adequadas, e crónica refratária quando preenche simultaneamente os critérios de ambas. Entende-se como tratamento adequado o tempo durante o qual uma dose apropriada de medicação é administrada, sendo tipicamente considerado pelo menos dois meses (preferencialmente três) em dose ótima ou dose máxima tolerada - a menos que terminada antes, devido a efeitos secundários. Este conceito requer o controlo dos fatores favorecedores da cronificação já referidos. Os critérios de diagnóstico para a enxaqueca crónica refratária encontram-se expressos numa declaração de consenso elaborada por um grupo de especialistas da European Headache Federation (EHF) com o objetivo de melhorar a identificação destes doentes e contribuir para a seleção dos casos que beneficiem de terapêuticas inovadoras $^{9,57}$ (Tabela 4). São ainda requisitos para o diagnóstico de enxaqueca crónica refratária: a) exclusão de cefaleias secundárias com ressonância magnética crânio-encefálica; b) análises de sangue e líquido cefalorraquidiano (LCR) dentro dos valores normais incluindo a pressão do LCR; c) desintoxicação farmacológica (em ambulatório ou 
Tabela 4 - Critérios de diagnóstico de enxaqueca crónica refratária (EHF)
A. Enxaqueca crónica - sem uso excessivo de medicação
B. Uso de medicação profilática em doses adequadas durante pelo menos 3 meses com cada fármaco
C. Ausência de efeito (ou contraindicações para o uso) de pelo menos 3 fármacos de cada um dos seguintes grupos de medicações preventivas nas doses recomendadas:
a. Beta-bloqueantes
Propranolol até $240 \mathrm{mg} / \mathrm{d}$
Metoprolol até $200 \mathrm{mg} / \mathrm{d}$
Atenolol até $100 \mathrm{mg} / \mathrm{d}$
Bisoprolol até $10 \mathrm{mg} / \mathrm{d}$
b. Anticonvulsivantes
Valproato de sódio até $1,5 \mathrm{~g} / \mathrm{d}$
Topiramato até $200 \mathrm{mg} / \mathrm{d}$
c. Antidepressivos tricíclicos
Amitriptilina até $75 \mathrm{mgxd}$
d. Outros
Flunarizina até $10 \mathrm{mg} / \mathrm{d}$
Candesartan até $16 \mathrm{mg} / \mathrm{d}$
e. Toxina botulínica $A$
155 - 195 U segundo protocolo PREEMP
D. Tratamento psiquiátrico adequado ou de outras comorbilidades a efetuar por um grupo multidisciplinar, se disponível.

internamento hospitalar) com recurso a fármacos orais ou intravenosos ou, em certos casos, apenas com aconselhamento. ${ }^{58}$

Esta forma de cefaleia é a mais grave e incapacitante, acarretando uma enorme perturbação da qualidade de vida e sérias implicações socioeconómicas. Grande parte destes doentes trabalham em tempo parcial e têm salários baixos, ou estão desempregados e com subsídio da segurança social, encontram-se sobrecarregados com despesas acrescidas devido ao consumo de grandes quantidades de fármacos, geralmente analgésicos, e recorrem com maior frequência aos cuidados médicos e aos serviços de urgência, representando cerca de $3 \%$ do total de visitas. ${ }^{58}$

Por definição, a enxaqueca refratária é resistente aos tratamentos farmacológicos, quer agudos, quer profiláticos. Neste contexto, restam como alternativas as terapêuticas mais recentes ou emergentes, nomeadamente a toxina botulínica, os anticorpos monoclonais contra o CGRP ou seus recetores, e os neuroestimuladores. Dado que os primeiros já foram mencionados apresentaremos aqui apenas os dados da neuroestimulação. ${ }^{57,58}$

A neuroestimulação pretende interferir com os sistemas de dor e a plasticidade sináptica. Pode classificar-se em não invasiva (ex: estimulação dos nervos supraorbitário ou vago, elétrica transcutânea ou magnética transcraniana) e invasiva (ex: estimulação cerebral profunda, do grande nervo occipital ou do gânglio esfenopalatino).

Os métodos não invasivos apresentam custos muito mais baixos sem os riscos inerentes à intervenção cirúrgica, podendo ainda ser usados como tratamento agudo da crise e como coadjuvantes da terapêutica farmacológica, como acontece por exemplo na estimulação percutânea do nervo supraorbitário.

Os métodos invasivos requerem intervenção cirúrgica para a colocação de implantes extracranianos ou intracranianos (estimulação cerebral profunda), com os riscos ine- rentes tais como infeções ou hemorragias, deslocação dos elétrodos, necessidade de renovação da fonte de energia (bateria), alteração do local da dor em caso de implante unilateral; perda de eficácia, para além do custo dos tratamentos. Devido aos seus riscos, os métodos invasivos destinam-se apenas ao tratamento de cefaleias primárias refratárias, nomeadamente, formas cronicas de cefaleia em salvas e enxaqueca, quando praticada em centros de cuidados terciários de cefaleias.

A evidência científica destes métodos, baseada em ensaios clínicos aleatorizados e controlados, é limitada, pelo que a EHF produziu uma Declaração de Consenso sobre a utilização da neuromodelação no tratamento das cefaleias crónicas refratárias e elaborou recomendações para futuros estudos sobre estes tratamentos. ${ }^{57}$ Assim, constitui requisito fundamental que a aplicação de um neuroestimulador seja considerado apenas quando as terapêuticas farmacológicas e comportamentais falharam e seja excluída a cefaleia por uso excessivo de medicação. O nível de eficácia desejável será uma redução de $50 \%$ no número de dias de cefaleias (embora possa ser admissível apenas $30 \%$ nas formas crónicas intratáveis). ${ }^{58}$

\section{CONCLUSÃO}

Nos indivíduos portadores de enxaqueca a frequência e severidades das crises variam ao longo do tempo, condicionadas por diversos fatores hormonais, sazonais, sociais ou psicológicos.

Nos períodos de agravamento, os doentes recorrem a medicação com maior frequência e quantidade. $O$ uso repetido desses medicamentos resulta em tolerância e habituação, o que favorece a transformação em enxaqueca crónica e posteriormente o desenvolvimento de uma nova forma de cefaleia - geralmente mais severa e incapacitante - com caraterísticas semelhantes à enxaqueca: a cefaleia por uso excessivo de medicação. A progressiva perda de 
eficácia dos analgésicos, simples ou compostos, e dos triptanos, torna a enxaqueca crónica refratária muito incapacitante e de muito difícil controlo. Para o tratamento destes doentes, que apresentam grande sofrimento e incapacidade funcional estão disponíveistécnicas de tratamento não farmacológico específicas - como por exemplo a utilização de neuroestimuladores - e farmacológico, tais como a toxina botulínica ou os anticorpos monoclonais contra o CGRP ou os seus recetores. Esta última constitui uma nova e promissora oportunidade terapêutica traduzindo poupanças relevantes de custos económicos e sociais.

\section{PROTECÇÃO DE PESSOAS E ANIMAIS}

Os autores declaram que os procedimentos seguidos estavam de acordo com os regulamentos estabelecidos pelos responsáveis da Comissão de Investigação Clínica e Ética e de acordo com a Declaração de Helsínquia da
Associação Médica Mundial.

\section{CONFIDENCIALIDADE DOS DADOS}

Os autores declaram ter seguido os protocolos do seu centro de trabalho acerca da publicação de dados.

\section{CONFLITO DE INTERESSES}

Elsa Parreira é consultora da Allergan, Linde e Novartis. Recebeu honorários por palestras e apoio para congressos da Angelini, Bial, Novartis, Allergan, Vitalaire, Linde e Teva. Isabel Luzeiro é consultora e recebeu honorários por palestras da Allergan, Novartis e Esai. JM Pereira Monteiro é consultor da Merck e Novartis.

\section{FONTES DE FINANCIAMENTO}

Os autores receberam da Novartis honorários pelo aconselhamento médico prestado.

\section{REFERÊNCIAS}

1. Woldeamanuel $Y$, Cowan R. Migraine affects 1 in 10 people worldwide featuring recent rise: $A$ systematic review and metanalysis of community-based studies involving 6 million participants. J Neurol Sci. 2017;372:307-15.

2. Stovner L, Andree C. Prevalence of headache in Europe: a review for the Eurolight project. J Headache Pain. 2010;11:289-99.

3. Stovner L, Hagen K, Jensen R, Katsarava Z, Lipton R, Scher Al, et al. The global burden of headache: a documentation of headache prevalence and disability worldwide. Cephalalgia. 2007;27:193-210.

4. GBD 2016 Disease and Injury Incidence and Prevalence Collaborators. Global, regional, and national incidence, prevalence, and years lived with disability for 328 diseases and injuries for 195 countries, 19902016: a systematic analysis for the Global Burden of Disease Study 2016. Lancet. 2017;390:1211-59.

5. Vuralli D, Ayata C, Bolay $\mathrm{H}$. Cognitive dysfunction and migraine. J Headache Pain. 2018;15;19:109.

6. Gil-Gouveia R, Martins I. Clinical description of attack-related cognitive symptoms in migraine: a systematic review. Cephalalgia. 2018;38:133550.

7. Lipton R, Bigal M, Diamond M, Freitag F, Reed M, Stewart W, et al. Migraine prevalence, disease burden, and the need for preventive therapy. Neurology. 2007;68:343-9.

8. Steiner T, Stovner J, Vos T, Jensen R, Katsarava K. Migraine is the first cause of disability under 50s: will health politicians now take notice? J Headache Pain. 2018;19:17.

9. Headache Classification Committee of the International Headache Society. The International Classification of Headache Disorders, $3^{\text {rd }}$ edition. Cephalalgia. 2018;38:1-211.

10. Buse D, Manack A, Fanning K, Serrano D, Reed M, Turkel C, et al. Chronic migraine prevalence, disability, and sociodemographic factors: results from the American Migraine Prevalence and Prevention Study. Headache. 2012;52:1456-70.

11. Natoli J, Manack A, Dean B, Butler Q, Turkel C, Stovner L, et al. Global prevalence of chronic migraine: a systematic review. Cephalalgia. 2009;30:599-609.

12. Manack A, Buse D, Lipton R. Chronic migraine: epidemiology and disease burden. Curr Pain Headache Rep. 2011;15:70-8.

13. Scher A, Midgette L, Lipton R. Risk factors for headache chronification. Headache. 2008;48:16-25.

14. Scher A, Stewart W, Ricci J, Lipton R. Factors associated with the onset and remission of chronic daily headache in a population-based study. Pain. 2003;106:81-9.

15. Buse D, Silberstein S, Manack A, Papapetropoulos S, Lipton R. Psychiatric comorbidities of episodic and chronic migraine. J Neurol. 2013;260:1960-9.

16. Buse D, Manack A, Serrano D, Turkel C, Lipton R. Sociodemographic and comorbidity profiles of chronic migraine and episodic migraine sufferers. J Neurol Neurosurg Psychiatry. 2010;81:428-32.

17. Adams A, Serrano D, Buse D, Reed M, Marske V, Fanning KM, et al.

The impact of chronic migraine: The Chronic Migraine Epidemiology and Outcomes (CaMEO) Study methods and baseline results. Cephalalgia. 2015;35:563-78.

18. Schwedt T. Chronic migraine. BMJ. 2014;24;348.

19. Bigal $M$, Serrano $D$, Reed M, Lipton $R$. Chronic migraine in the population: burden, diagnosis, and satisfaction with treatment. Neurology. 2008;71:559-66.

20. Bashir A, Lipton R, Ashina S, Ashina M. Migraine and structural changes in the brain: a systematic review and meta-analysis. Neurology. 2013;81:1260-8.

21. Schwedt T, Chong C, Wu T, Gaw N, Fu Y, Li J. Accurate classification of chronic migraine via brain magnetic resonance imaging. Headache. 2015;55:762-77.

22. Neeb L, Bastian K, Villringer K, Israel K, Reuter U, Fiebach J. Structural gray matter alterations in chronic migraine: implications for progressive disease? Headache. 2016;57:400-6.

23. Cernuda-Morollon E, Larrosa D, Moris G, Ramon C, Pascual J. Increased levels of CGRP in peripheral blood in women with chronic migraine: a reliable biological marker. J Headache Pain. 2013;14:P104.

24. Marques I, Sousa L. Abordagem das cefaleias diárias. In: Martins IP, editor. Cefaleias. Lisboa: Lidel, 2015. p 129-36.

25. Yalın Ö, Uluduz D, Özge A, Sungur M, Selekler M, Siva A. Phenotypic features of chronic migraine Osman. J Headache Pain. 2016;17:26.

26. Cho S, Song T, Chu M. Treatment update of chronic migraine. Curr Pain Headache Rep. 2017;21:26.

27. Silberstein S, Holland S, Freitag F, Dodick D, Argoff C, Ashaman E, et al. Evidence-based guideline update: pharmacologic treatment for episodic migraine prevention in adults: report of the quality standards subcommittee of the American Academy of Neurology and the American Headache Society. Neurology. 2012;78:1337-45.

28. Yurekli V, Akhan G, Kutluhan S, Uzar E, Koyuncuoglu H, Gultekin F. The effect of sodium valproate on chronic daily headache and its subgroups. J Headache Pain. 2008;9:37-41.

29. Diener H, Bussone G, Van Oene J, Lahaye M, Schwalen S, Goadsby P. Topiramate reduces headache days in chronic migraine: a randomized, double-blind, placebo-controlled study. Cephalalgia. 2007;27:814-23.

30. Silberstein S, Lipton R, Dodick D, Freitag F, Ramadan N, Mathew N, et al. Topiramate Chronic Migraine Study Group. Efficacy and safety of topiramate for the treatment of chronic migraine: a randomized, doubleblind, placebo-controlled trial. Headache. 2007;47:1398-408.

31. Hepp Z, Dodick D, Varon S, Gillard P, Hansen R, Devine E. Adherence to oral migraine-preventive medications among patients with chronic migraine. Cephalalgia. 2015;35:478-88.

32. Aurora S, Winner P, Freeman M, Spierings E, Heiring J, DeGryse R, et al. Onabotulinumtoxin A for treatment of chronic migraine: pooled analyses of the 56-week PREEMPT clinical program. Headache. 2011;51:1358-7.

33. Edvinsson L, Haanes K, Warfvinge K, Krause D. CGRP as the target of new migraine therapies - successful translation from bench to clinic. Nat Rev Neurol. 2018;14:338-50. 
34. Tepper S. History and review of anti-calcitonin gene-related peptide (CGRP) therapies: from translational research to treatment. Headache. 2018;58:S217-97

35. Tepper S, Ashina M, Reuter U, Brandes J, Doležil D, Silberstein S, et al. Safety and efficacy of erenumab for preventive treatment of chronic migraine: a randomised, double-blind, placebo-controlled phase 2 trial. Lancet Neurology. 2017;16:425-34.

36. Smith J, Dodick D, Goadsby P, Silberstein S, Lipton R, Hirman J. Randomized, double-blind, placebo-controlled trial of ALD403 (eptinezumab), an anti-CGRP monoclonal antibody for the prevention of chronic migraine. Headache. 2017;57:130.

37. Mitsikostas D, Reuter U. Calcitonin gene-related peptide monoclonal antibodies for migraine prevention: comparisons across randomized controlled studies. Curr Opin Neurol. 2017;30:272-80.

38. Gouveia R, Parreira E. Utilização de anticorpos monoclonais na terapêutica da enxaqueca. A revolução terapêutica há muito esperada? Sinapse. 2018;18:50-65.

39. Manack A, Buse D, Serrano D, Turkel C, Lipton R. Rates, predictors, and consequences of remission from chronic migraine to episodic migraine. Neurology. 2011;76:711-8.

40. Westergaard M, Munksgaard S, Bendtsen L, Jensen R. Medicationoveruse headache: a perspective review. Ther Adv Drug Saf. 2016;7:147-58.

41. Steiner T, Stovner L, Katsarava Z, Lainez J, Lampl C, Lantéri-Minet M, et al. The impact of headache in Europe: principal results of the Eurolight project. J Headache Pain. 2014;15:1-11.

42. Linde M, Gustavsson A, Stovner L, Steiner T, Barré J, Katsarava Z, et al. The cost of headache disorders in Europe: The Eurolight Project. Eur J Neurol. 2012;19:703-11.

43. Diener H, Limmroth V. Medication-overuse headache: a worldwide problem. Lancet Neurol. 2004;3:475-83

44. Radat F, Lanteri-Minet $M$. What is the role of dependence-related behavior in medication-overuse headache? Headache. 2010;50:1597611.

45. Lundqvist C, Grande R, Aaseth K, Russell M. Dependence scores predict prognosis of medication overuse headache: a prospective cohort from the Akershus study of chronic headache. Pain. 2012;153:682-6.

46. Hagen K, Linde M, Steiner T, Stovner L, Zwart J. Risk factors for medication-overuse headache: an 11-year follow-up study. The NordTrøndelag Health Studies. Pain. 2012;153:56-61.

47. Rossi P, Di Lorenzo C, Faroni J, Cesarino F, Nappi G. Advice alone vs. structured detoxification programmes for medication overuse headache: a prospective, randomized, open-label trial in transformed migraine patients with low medical needs. Cephalalgia. 2006;26:1097-105.

48. Supornsilpchai W, Le Grand S, Srikiatkhachorn A. Involvement of pronociceptive 5-HT2A receptor in the pathogenesis of medication-overuse headache: research submission. Headache. 2010;50:185-97.

49. De Felice M, Porreca F. Opiate-induced persistent pronociceptive trigeminal neural adaptations: Potential relevance to opiate-induced medication overuse headache. Cephalalgia. 2009;29:1277-84.

50. Grazzi L, Chiapparini L, Ferraro S, Usai S, Andrasik F, Mandelli M, et al. Chronic migraine with medication overuse pre-post withdrawal of symptomatic medication: clinical results and fMRI correlations. Headache. 2010;50:998-1004.

51. Ferraro S, Grazzi L, Mandelli M, Aquino D, Fiorwe D, Usai S, et al. Pain processing in medication overuse headache: a functional magnetic resonance imaging (fMRI) study. Pain Med. 2012;13:255-62.

52. Riederer $F$, Marti M, Luechinger R, Lanzenberger R, Meyenburg J, Gantenbein A, et al. Grey matter changes associated with medicationoveruse headache: Correlations with disease related disability and anxiety. World J Biol Psychiatry. 2012;13:517-25.

53. Munksgaard S, Bendtsen L, Jensen R. Detoxification of medicationoveruse headache by a multidisciplinary treatment programme is highly effective: a comparison of two consecutive treatment methods in an open-label design. Cephalalgia. 2012;32:834-44.

54. Rossi P, Faroni J, Nappi G. Medication overuse headache: predictors and rates of relapse in migraine patients with low medical needs. A 1-year prospective study. Cephalalgia. 2008;28:1196-200.

55. Probyn K, Bowers H, Caldwell F, Mistry D, Underwood M, Matharu M, et al. Prognostic factors for chronic headache. Neurology. 2017;89:291 301.

56. Schulman E, Lake A, Goadsby P, Peterlin B, Siegel S, Markley H, et al Defining refractory migraine and refractory chronic migraine: proposed criteria from the Refractory Headache Special Interest Section on the American Headache Society. Headache. 2008;48:778-82.

57. Marteletti P, Katsarava Z, Lampi C, Magis D, Bendtsen L, Negro A, et al. Refractory chronic migraine: a consensus statement on clinical definition from the European Headache Federation. J Headache Pain. 2014;15:47-56.

58. Zagami AS. Treatment of the patient with refractory headache. Curr Pain Headache Rep. 2018;22:23. 